\title{
(GIGA)bYte
}

\section{Utilizing a chromosomal-length genome assembly to annotate the Wnt signaling pathway in the Asian citrus psyllid, Diaphorina citri}

Chad Vosburg', ${ }^{1,2}$ Max Reynolds ${ }^{1}$, Rita Noel $^{1}$, Teresa Shippy ${ }^{3}$, Prashant S. Hosmani ${ }^{4}$, Mirella Flores-Gonzalez ${ }^{4}$, Lukas A. Mueller ${ }^{4}$, Wayne B. Hunter ${ }^{5}$, Susan J. Brown ${ }^{3}$, Tom D’Elia ${ }^{1}$ and Surya Saha ${ }^{4,6, *}$

1 Indian River State College, Fort Pierce, FL 34981, USA

2 Department of Plant Pathology and Environmental Microbiology, Pennsylvania State University, University Park, PA 16802, USA

3 KSU Bioinformatics Center, Division of Biology, Kansas State University, Manhattan, KS 66506, USA

4 Boyce Thompson Institute, Ithaca, NY 14853, USA

5 USDA-ARS, US Horticultural Research Laboratory, Fort Pierce, FL 34945, USA

6 Animal and Comparative Biomedical Sciences, University of Arizona, Tucson, AZ 85721, USA

\section{ABSTRACT}

The Asian citrus psyllid, Diaphorina citri, is an insect vector that transmits Candidatus Liberibacter asiaticus, the causal agent of the Huanglongbing (HLB), or citrus greening disease. This disease has devastated Florida's citrus industry, and threatens California's industry as well as other citrus producing regions around the world. To find novel solutions to the disease, a better understanding of the vector is needed. The $D$. citri genome has been used to identify and characterize genes involved in Wnt signaling pathways. Wnt signaling is utilized for many important biological processes in metazoans, such as patterning and tissue generation. Curation based on RNA sequencing data and sequence homology confirms 24 Wnt signaling genes within the $D$. citri genome, including homologs for beta-catenin, Frizzled receptors, and seven Wnt-ligands. Through phylogenetic analysis, we classify $D$. citri Wnt ligands as $W g / W n t 1$, Wnt5, Wnt6, Wnt7, Wnt10, Wnt11, and WntA. The D. citri version 3.0 genome with chromosomal length scaffolds reveals a conserved Wnt1-Wnt6-Wnt10 gene cluster with a gene configuration like that in Drosophila melanogaster. These findings provide greater insight into the evolutionary history of $D$. citri and Wnt signaling in this important hemipteran vector. Manual annotation was essential for identifying high quality gene models. These gene models can be used to develop molecular systems, such as CRISPR and RNAi, which target and control psyllid populations to manage the spread of HLB. Manual annotation of Wnt signaling pathways was done as part of a collaborative community annotation project.

suryasaha@cornell.edu

Published by GigaScience Press.

Preprint submitted at https:

//doi.org/10.1101/2020.09.21.306100

Subjects Genetics and Genomics, Animal Genetics, Bioinformatics

This is an Open Access article distributed under the terms of the Creative Commons Attribution License (http://creativecommons.org/ licenses/by/4.0/), which permits unrestricted reuse, distribution, and reproduction in any medium, provided the original work is properly cited.

Gigabyte, 2021, 1-15

\section{DATA DESCRIPTION}

\section{Introduction}

Diaphorina citri (NCBI:txid121845) is the insect vector of Huanglongbing (HLB), or citrus greening disease, which has devastated global citrus production [1, 2]. HLB management is heavily based on controlling the spread of $D$. citri. To better understand the insect's 
biology, the $D$. citri genome has been manually annotated to curate accurate gene model predictions. Accurate gene models can be used to develop novel insect control systems that utilize molecular therapeutics such as CRISPR (clustered regularly interspaced short palindromic repeats) and RNA interference (RNAi) to control the spread of $D$. citri [3, 4]. These molecular therapeutics would be gene-specific, thus would reduce reliance on broad-spectrum insecticides that have given rise to resistant $D$. citri populations [5-7].

\section{Context}

Here, we report $D$. citri genes involved in both canonical and noncanonical Wnt signaling. Wnt signaling is important for many biological processes in metazoans, such as patterning, cell polarity, tissue generation, and stem cell maintenance [8-10]. In the model insects Drosophila melanogaster and Tribolium castaneum, knockout and knockdown of Wnt ligands and other Wnt signaling components have detrimental effects on embryo development and adult homeostasis [11-16]. Wnt signaling components could therefore be effective knockout targets to limit the spread of $D$. citri, thus reducing HLB incidence. We curated a comprehensive repertoire of Wnt signaling genes in $D$. citri. Twenty-four gene models corresponding to canonical and noncanonical Wnt signaling genes have been annotated, including seven Wnt ligands, three frizzled homologs, arrow, armadillo/beta-catenin, and receptor tyrosine kinases ROR and doughnut. We were unable to find Wnt8/D, Wnt9, and Wnt16 as well as Wnt2-4, which have been lost in insects. The mechanisms of Wnt signaling appear to be mostly conserved and comparable to those found in D. melanogaster (Table 1). A model for canonical Wnt signaling in D. citri based on curated genes is shown (Figure 1). This is an important first step towards understanding critical biological processes that might be targeted to control the spread of $D$. citri, and may provide broader insights into the mechanisms of Wnt signaling in this important hemipteran vector.

\section{METHODS}

We used the psyllid genome curation workflow used for community annotation (Figure 2) [17].

To summarize, orthologous protein sequences for Wnt pathway genes were collected from the National Center for Biotechnology Information (NCBI) (RRID:SCR_006472) protein database [18] and used to BLAST (RRID:SCR_004870) search the D. citri MCOT transcriptome database [19]. The MCOT transcriptome is a transcriptome assembly utilizing Maker (RRID:SCR_005309), Cufflinks (RRID:SCR_014597), Oases (RRID:SCR_011896), and Trinity (RRID:SCR_013048) pipelines to provide a comprehensive set of predicted gene models. High-scoring MCOT models (accessions available in Table 2) were then searched on the NCBI protein database using NCBI BLAST to confirm the viability of the predicted MCOT models. The high-scoring MCOT models that had promising NCBI search results were used to search the $D$. citri genome. Genome regions containing computationally predicted gene models with high sequence identity to the query sequence from the MCOT transcriptome were investigated within JBrowse (RRID:SCR_001004). Gene models were modified using the Apollo (RRID:SCR_001936) gene annotation platform, based on mapped DNA-Seq, RNA-Seq, Iso-Seq, orthologous proteins, and other lines of evidence to edit and confirm manual annotations and gene structure. The gene models were analyzed with NCBI BLAST to assess 


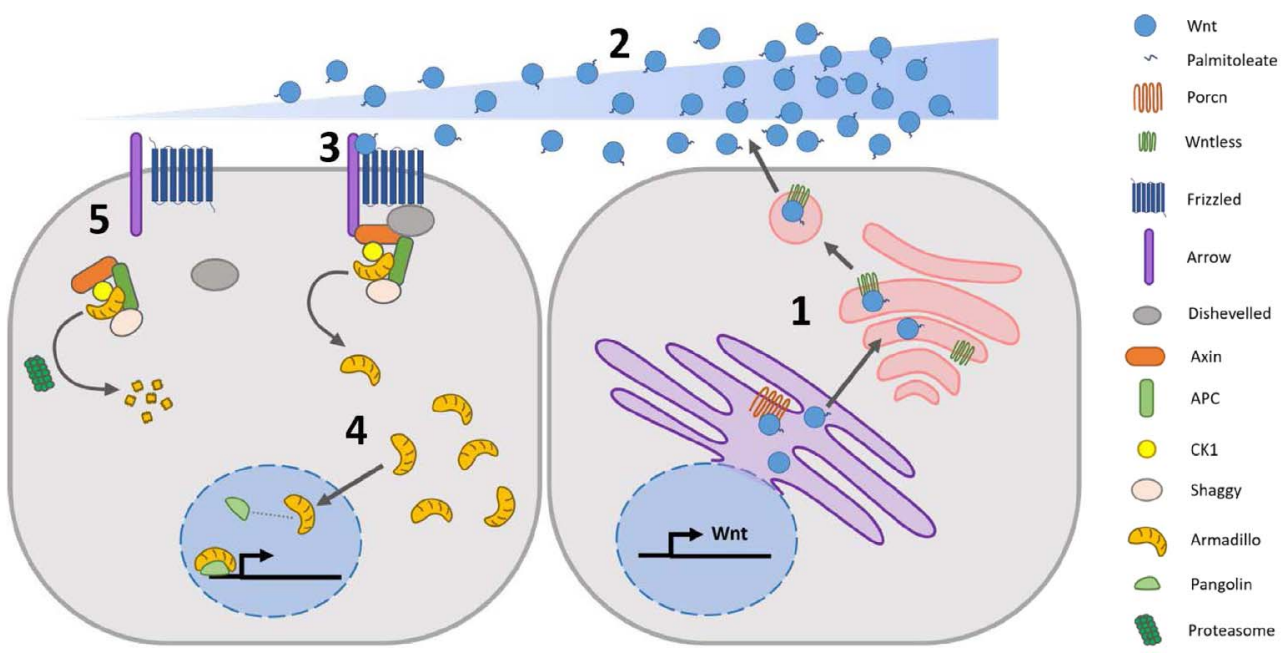

Figure 1. Theoretical model of canonical Wnt signaling cascade in D. citri based on curated genes. (1) Wnt is secreted. (2) Wnt concentration gradient forms. (3) Wnt binds to Frizzled and releases Armadillo. (4) Armadillo migrates into the nucleus, associates with transcription factor Pangolin, and regulates gene expression. (5) Armadillo is degraded in the absence of Wnt.

Table 1. Summary of gene copy numbers in various model insect species, including Diaphorina citri. Wnt pathway ortholog numbers in five different insect species. Drosophila melanogaster, Apis mellifera, Tribolium castaneum, and Acyrthosiphon pisum copy numbers were determined using Flybase, OrthoDB, NCBI Genbank, Uniprot, and several other publications [15, 20-22]. Diaphorina citri numbers represent the number of manually annotated genes in the $D$. citri v3.0 genome.

\begin{tabular}{|cccccc|}
\hline Gene & $\begin{array}{c}\text { Drosophila } \\
\text { melanogaster }\end{array}$ & Apis mellifera & $\begin{array}{c}\text { Tribolium } \\
\text { castaneum }\end{array}$ & $\begin{array}{c}\text { Acyrthosiphon } \\
\text { pisum }\end{array}$ & $\begin{array}{c}\text { Diaphorina } \\
\text { citri v3 }\end{array}$ \\
\hline Wnt1 & 1 & 1 & 1 & 1 & 1 \\
\hline Wnt5 & 1 & 1 & 1 & 1 & 1 \\
\hline Wnt6 & 1 & 1 & 1 & 0 & 1 \\
\hline Wnt7 & 1 & 1 & 1 & 1 & 1 \\
\hline Wnt8/D & 1 & 0 & 1 & 0 & 0 \\
\hline Wnt9 & 1 & 0 & 1 & 0 & 1 \\
\hline Wnt10 & 1 & 1 & 1 & 0 & 1 \\
\hline Wnt11 & 0 & 1 & 1 & 1 & 0 \\
\hline Wnt16 & 0 & 0 & 0 & 1 & 1 \\
\hline WntA & 0 & 1 & 1 & 1 & 1 \\
\hline Pangolin & 1 & 1 & 1 & 1 & 1 \\
\hline Armadillo & 1 & 1 & 2 & 2 & 1 \\
\hline Wntless & 1 & 1 & 1 & 1 & 1 \\
\hline Porcupine & 1 & 1 & 1 & 1 & 1 \\
\hline Derailed & 2 & 1 & 0 & 1 & 1 \\
\hline Doughnut & 1 & 1 & 1 & 1 & 3 \\
\hline Arrow & 1 & 1 & 1 & 1 & 2 \\
\hline Frizzled & 4 & 2 & 3 & 2 & 1 \\
\hline ROR & 2 & 2 & 3 & 2 & 1 \\
\hline Dishevelled & 1 & 1 & 1 & 1 & 1 \\
\hline Shaggy & 1 & 1 & 1 & 2 & 1 \\
\hline Axin & 1 & 1 & 1 & 1 & 1 \\
\hline ck1-gamma & 1 & 1 & 1 & 1 & \\
\hline Apc & 2 & 1 & 1 & 1 & \\
\hline & & & & & \\
\hline
\end{tabular}

their completeness. MUSCLE (RRID:SCR_011812) multiple sequence alignments of the $D$. citri gene model sequences and orthologous sequences were created through MEGA7 


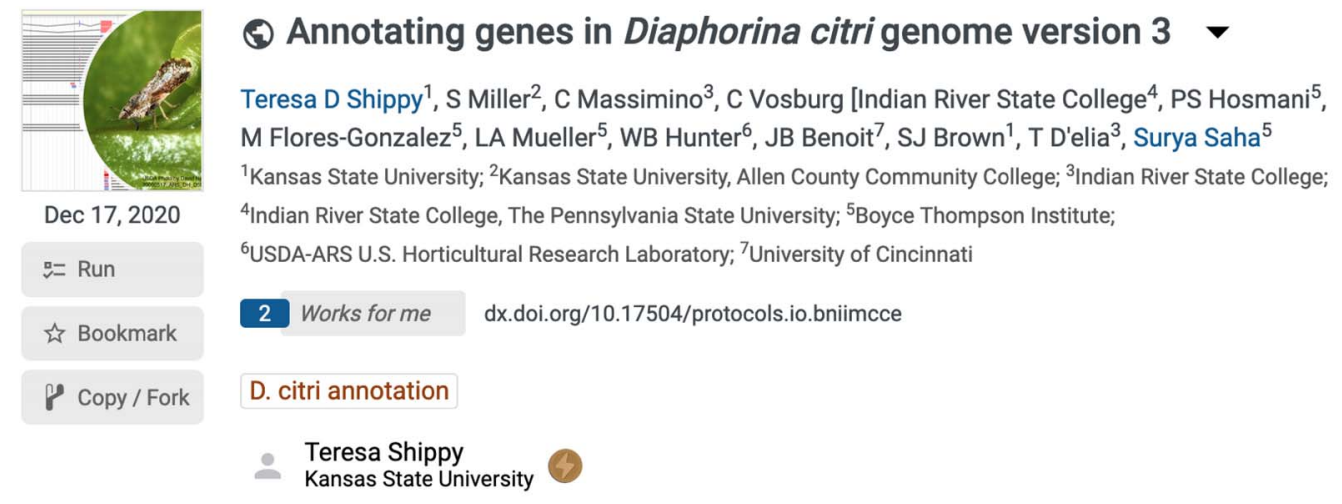

Figure 2. Protocol for psyllid genome curation [17]. https://www.protocols.io/widgets/doi?uri=dx.doi.org/10. 17504/protocols.io.bniimcce

(RRID:SCR_000667) [23]. Neighbor-joining trees were constructed using MEGA7 with p-distance for determining branch length and 1000 bootstrapping replications to measure the precision of branch placement. In special cases, phylogenetic analysis in conjunction with NCBI BLAST scores was used to properly name and characterize the manually annotated gene models.

RNA-seq data from whole body adults and nymphs raised on $C$. medica and $C$. sinensis are available from NCBI BioProject PRJNA609978. We used proteins from Drosophila melanogaster (Dm) [24], Tribolium castaneum [25], Bombyx mori [26], Apis mellifera [27], Nasonia vitripennis [28], Acyrthosiphon pisum [29], Nilaparvata lugens [30, 31], Sipha flava [32], Halyomorpha halys [33], Cimex lectularius [34], Aedes aegypti [35], Anopheles gambiae [36], Branchiostoma floridae [37], Penaeus vannamei [38], Folsomia candida [39], Spodoptera litura [40], Homo sapiens [32] and Oncopeltus fasciatus [34, 41].

\section{Data validation and quality control}

The loss of Wnt ligand genes is more common in insects than in other metazoans [20], which leads to a highly variable array of Wnt genes and Wnt signaling components from species to species [15, 21, 22, 42]. We performed a phylogenetic analysis to characterize the $D$. citri Wnt repertoire (Figure 3). The ortholog sequences used for this analysis were collected from the NCBI protein database [18]; see the 'Availability of Supporting Data and Materials' section for accession numbers. Seven $D$. citri Wnts were identified and classified as Wnt1 (also known as wingless), Wnt5, Wnt6, Wnt7, Wnt10, Wnt11, and WntA (Figures 3 and 4). In comparison, seven Wnt genes have been identified in D. melanogaster, nine in T. castaneum, and six in Acyrthosiphon pisum [22, 42]. The collection of Wnt genes found in D. citri is like that found in other insects, and no Wnt subfamilies have been identified as being unique to D. citri. Contrary to previous reports [43], D. citri does appear to possess a Wnt6 gene.

Wnt1, Wnt6, and Wnt10 typically occur close together in a highly conserved gene cluster [44, 45]. The chromosomal length genome assembly in v3.0 suggests that this cluster is also conserved in $D$. citri, located at a position between $26.4 \mathrm{Mb}$ (megabases) and 26.6 $\mathrm{Mb}$ on scaffold 4 (i.e. chromosome 4) [46]. In comparison, the Wnt1-6-10 cluster is located at a position between $7.30 \mathrm{Mb}$ and $7.38 \mathrm{Mb}$ on chromosome $2 \mathrm{~L}$ of $D$. melanogaster, and between $5.50 \mathrm{Mb}$ and $5.53 \mathrm{Mb}$ on linkage group 5 in $T$. castaneum. The only gene from this cluster 


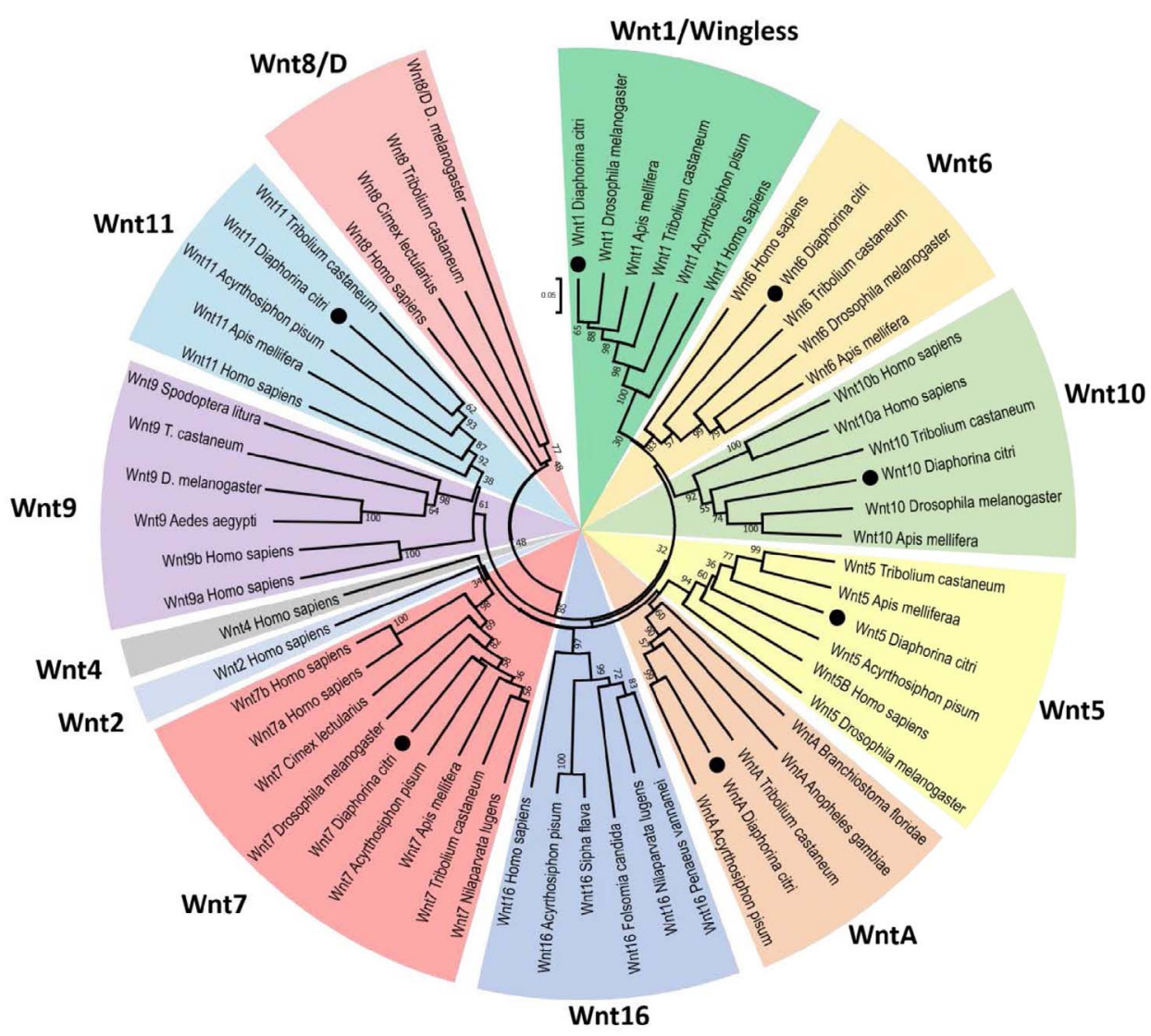

Figure 3. Neighbor-joining tree of Wnt protein sequences. Phylogenetic analysis was performed to categorize the seven $D$. citri Wnt genes (signified by dots). Wnt families are distinguished by clades and are color coded. Bootstrap values are based on 1000 replicates and values under 25 are removed. Ortholog sequences were collected from NCBI protein database [18]. Analysis was performed using MEGA7 [23].

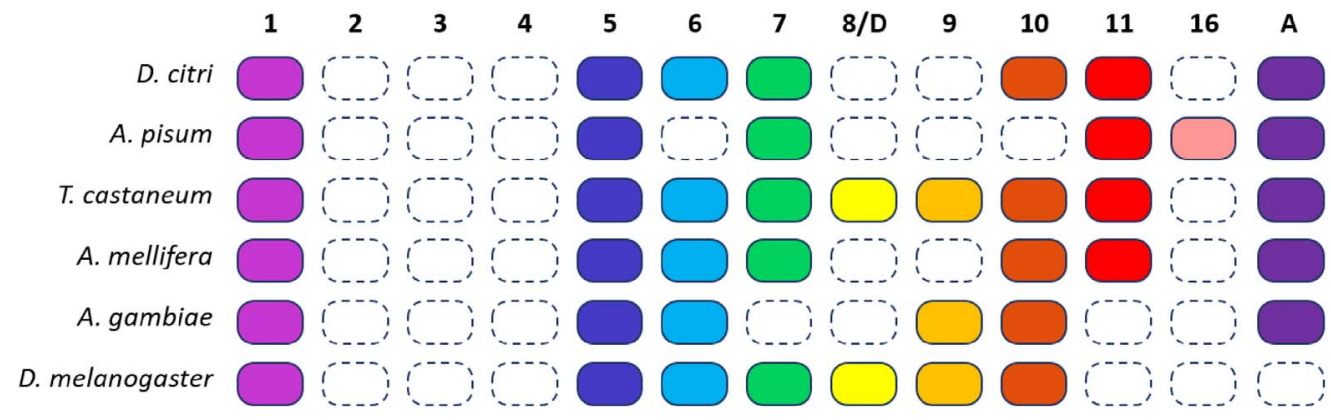

Figure 4. Wnt genes in six insects. A colored box indicates the presence of a Wnt subfamily (1-11, 16, and A) in that insect, while a white box indicates the loss of a subfamily. For example, all six species have Wnt 1 and Wnt5, none have Wnt2-4, and only A. pisum has Wnt16. Homologs of Wnt8 in T. castaneum and D. melanogaster are also referred to as WntD.

present in A. pisum is Wnt1, which is located on the $\mathrm{X}$ chromosome. The close phylogenetic relationship of Wnt1, Wnt6, and Wnt10 in D. citri (Figure 3) supports the hypothesis that this cluster is the result of an ancient duplication event, one that may predate the 


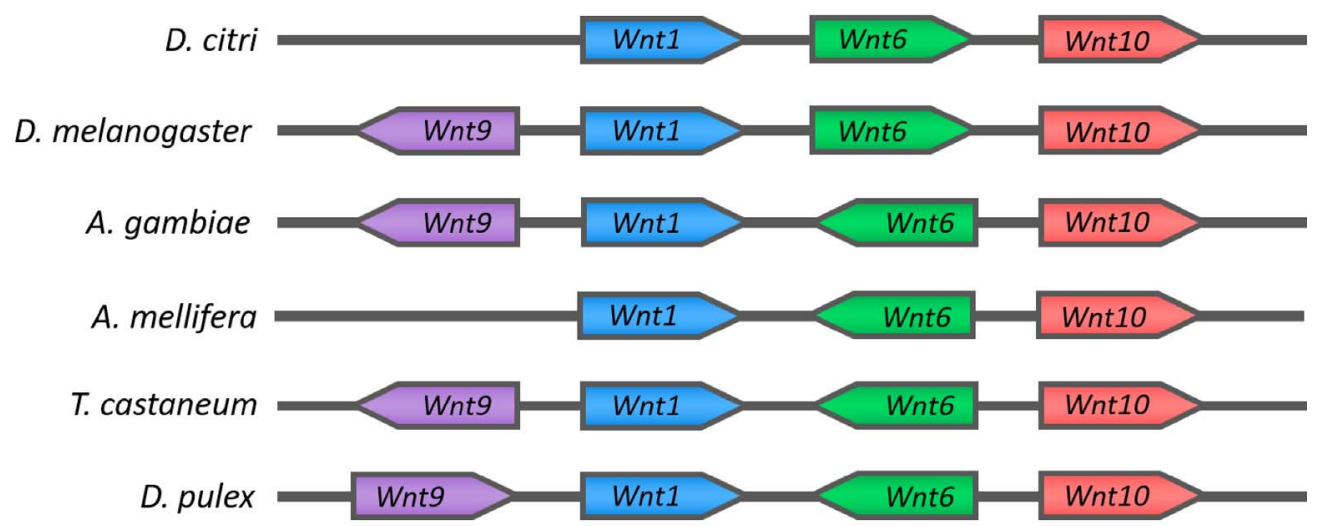

Figure 5. Wnt1-6-10 cluster comparison. Organization of the Wnt1-6-10 cluster in D. citri is similar to that of $D$. melanogaster and differs from what may be a basal arthropod gene arrangement seen in A. gambiae, T. castaneum, A. mellifera, and D. pulex. Gene lengths are not to scale.

divergence of cnidarians and bilaterians [45]. The orientation of these clustered D. citri Wnt genes is like that found in D. melanogaster and differs from what may be a basal arthropodal organization of Wnts found in species of Coleoptera, Hymenoptera, and Cladocera (Figure 5). Wnt9 is also associated with this gene cluster when present in the genome. However, as with A. pisum, Wnt9 was not found in the D. citri genome and appears to have been lost during evolution. A second Wnt cluster, Wnt5 and Wnt7, is also common among non-insect metazoans. This cluster is not seen in D. citri; however, D. citri Wnt5 and 7 are located relatively close to one another (within $220 \mathrm{~Kb}$ [kilobase pairs]) on scaffold 13 (i.e. chromosome 13).

The mechanisms that act to conserve these Wnt gene clusters are not well understood. In the basal metazoan Nematostella vectensis, clustered Wht genes do not exhibit similar expression patterns or Hox-like collinearity [44], and may not share regulatory elements. Whole body transcript expression data from egg, nymph, and adult stages [47] obtained from the Citrus Greening Expression Network (CGEN) [48] shows varying levels of expression among the clustered genes in different life stages of $D$. citri (Figure 6). However, it appears that Wnt 1 and 10 are similarly upregulated during embryonic psyllid development and downregulated during the adult stage. Similar transcript levels of Wnt1 and 6 are seen in the nymphal stage. This suggests there may be shared regulation dependent upon life stage. Furthermore, ordering within the clusters is subject to rearrangement (Figure 5) [42, 44]. This may indicate that gene directionality is not a factor in conserving this cluster. Our annotation findings support the hypothesis that the Wnt1-6-10 cluster is being preserved through either natural selection or an unknown mechanism. A better understanding of the regulatory hierarchy controlling Wnt expression might shed light on the significance of $W n t$ gene associations in the genome. Future characterization of the coding and noncoding regions surrounding these D. citri Wnts (e.g., tandem repeat analysis) could also provide more insight into the mechanisms causing Wnt duplication events.

The organization of the genomic reference sequence into chromosomal length scaffolds was essential for revealing $D$. citri gene clustering. Because of their shorter scaffold lengths, previous genome assemblies were often unsupportive in confirming the proximity of genes. 


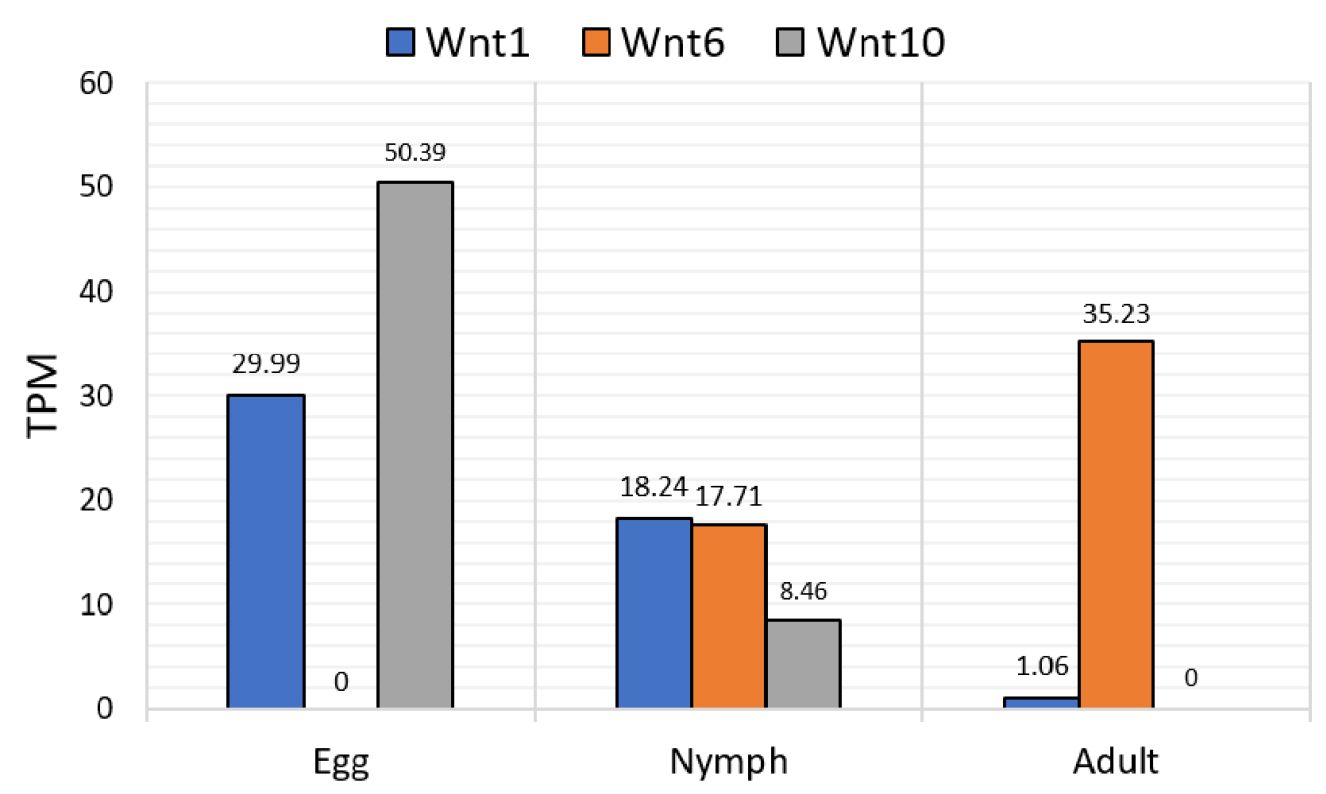

Figure 6. Transcript levels of clustered Wnt transcripts during different $D$. citri life stages. Whole body transcript expression data from egg, nymph, and adult stages [47] were collected from CGEN [48]. The psyllids were raised on Citrus macrophylla and were not infected with Candidatus Liberibacter asiaticus. Expression values shown in transcripts per million (TPM).

Genome v2.0 assembly errors had likely misrepresented the location of Wnt10, making it appear to be separated from Wnt1 and Wnt6. A complete Wnt1-6-10 cluster was found in the improved chromosome length assembly v3.0. Thus, the quality of the reference genome should be considered when performing phylogenetic studies.

Orthologs for Wnt2, Wnt3, Wnt4, Wnt8/D, Wnt9, and Wnt16 were not located in the $D$. citri genome. The close identity of certain Wnt subfamilies makes it difficult to distinguish between them; however, the loss of Wnt2-4 is expected because they are absent in all insects [20]. Apis mellifera and the hemipteran A. pisum have been reported to lack Wnt8/D. Perhaps this Wnt subfamily has been lost in the divergence from other insect groups [22]. Additionally, Wnt16 was not found in D. citri v3.0. This finding contrasts with the gene predictions of other hemipteran genomes available at NCBI, namely A. pisum, Sipha flava, and Nilaparvata lugens (Figure 3). Based on whole body RNA expression data collected from CGEN, Wnt6 has the highest average transcript levels of all the Wnt genes in both nymph and adult psyllids (Figure 7). The relatively high number of transcripts suggests that Wnt6 is important during both metamorphosis and adult stage homeostasis, and may be a good knockout target for molecular therapeutics. Transcript expression of Wnt6 in adults is mainly concentrated in the legs and thorax, averaging 102 transcripts per million (TPM) and 272 TPM, respectively. This is considerably higher than all other Wnt genes in these tissues, which only average between 0.26 and 3.00 TPM. It is unclear if other Wnts can be upregulated to compensate for the loss of Wnt6. Perhaps targeting multiple Wnt genes, or the mechanisms by which Wnt is secreted (i.e. Porcupine and Wntless), would be more disruptive to $D$. citri physiology.

Several receptors and co-receptors associated with canonical and noncanonical signaling have been identified (Table 2). Three paralogs for the Wnt receptor encoding frizzled have 


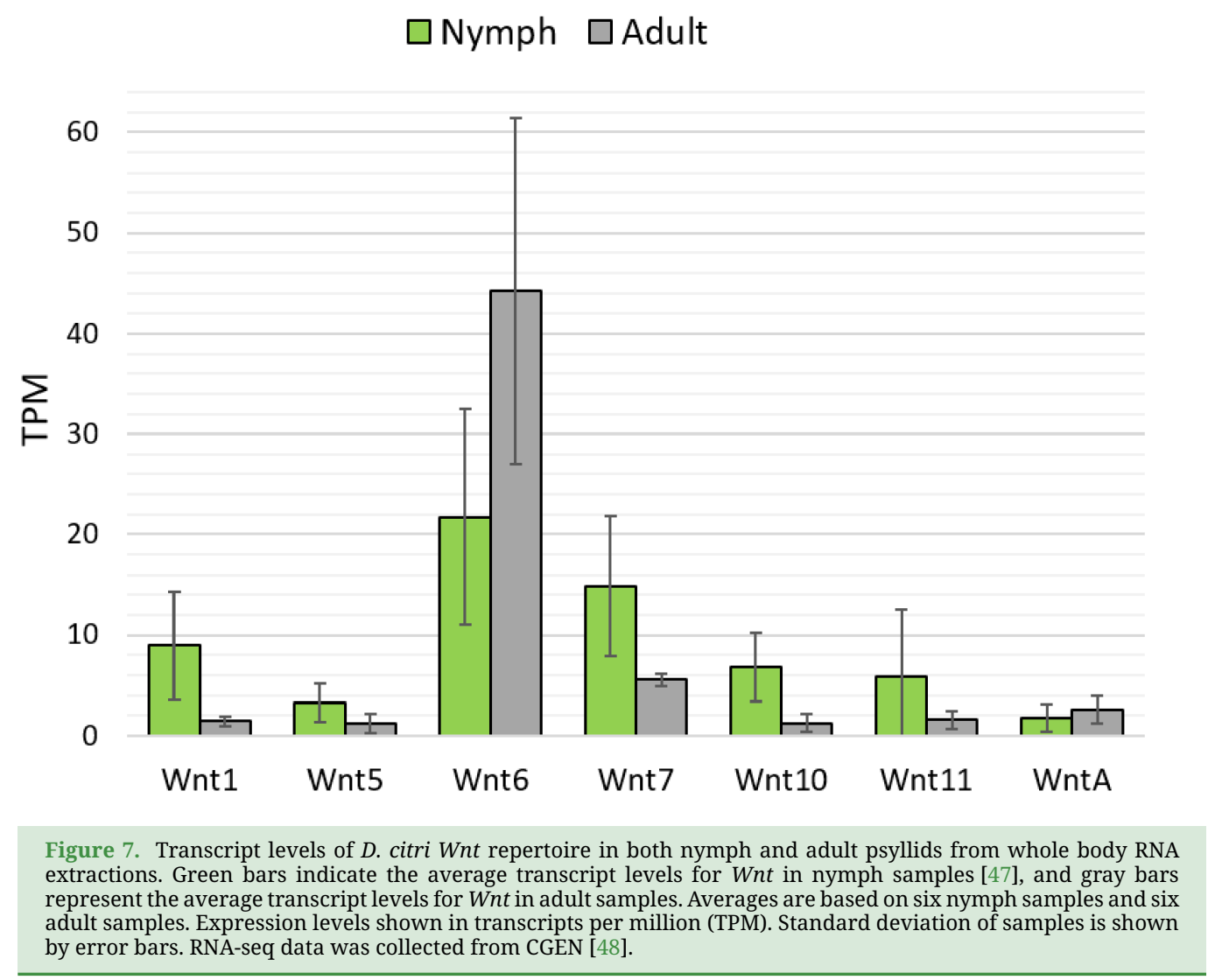

been found in $D$. citri. We classified and numerically designated $D$. citri's three frizzled genes based on how their encoded protein sequences form clades with $D$. melanogaster orthologs (Figure 8). Our analysis showed that D. citri, and other hemipterans such as Halymorpha halys and $N$. lugens, possess a Frizzled protein like that of D. melanogaster's Frizzled 3. Some hemipteran Frizzled orthologs form a distinct clade separate from the Dipteran sequences (Figure 8). The hemipteran clade suggests that these genes might belong to a different subfamily of Frizzled, maybe one specific to Hemiptera. However, this ortholog has not been reported in the A. pisum genome [22].

Orthologs for both ROR1 and ROR2 have been identified. Interestingly, ROR1 has two isoforms, the first of which contains an immunoglobulin (IG) domain that is lacking from isoform 2 (Figure 9). ROR1 isoform 2 (Dcitr05g14430.1.2) appears to average higher transcript levels in $D$. citri egg, nymph, and adult tissues than ROR1 isoform 1 (Dcitr05g14430.1.1) based on CGEN data (Figure 10). Many transcripts for isoform 2 were detected in the psyllid egg (Figure 10). This suggests that expression of isoform 2 may be important in the early developmental stages of $D$. citri.

\section{Conclusion}

Controlling the spread of $D$. citri is an important strategy for reducing the spread of HLB. With this study, we hope to provide a greater insight into $D$. citri biology, as well as accurate gene models that can be used in future research and applications. We have curated a comprehensive repertoire of $W n t$ signaling genes in $D$. citri. In total, 24 gene models 


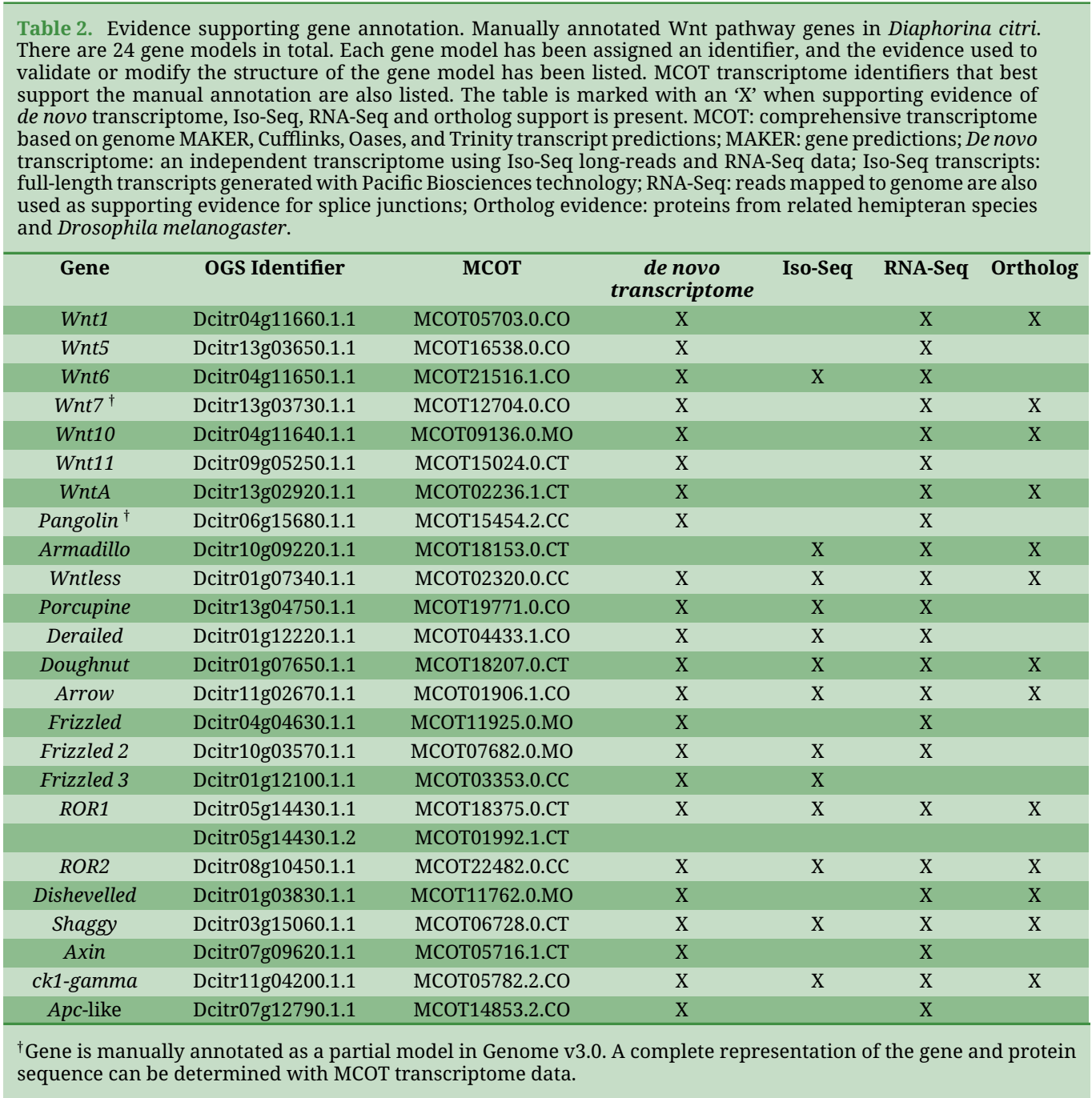

corresponding to canonical and noncanonical Wnt signaling have been annotated. The mechanisms of Wnt signaling appear to be mostly conserved and comparable to those found in D. melanogaster and other insects. These findings provide a greater insight into the evolutionary history of $D$. citri and Wnt signaling in this important hemipteran vector. Manual annotation and an improved genome assembly with chromosomal length scaffold were essential for identifying high quality gene models.

\section{REUSE POTENTIAL}

The manually curated genes will be included in the Citrus Greening Expression Network (CGEN) [48] as a part of the Official Gene Set version 3. This visualization tool is useful for understanding psyllid biology and comparative analysis because it contains public transcriptomics data for Diaphorina citri from various tissues, life stages, CLas infection levels and citrus hosts. Future work could utilize these gene models in developing CRISPR and RNAi systems that target and disrupt critical biological processes in $D$. citri, thus controlling the spread of HLB. This work was done as part of a collaborative community annotation project [49]. 


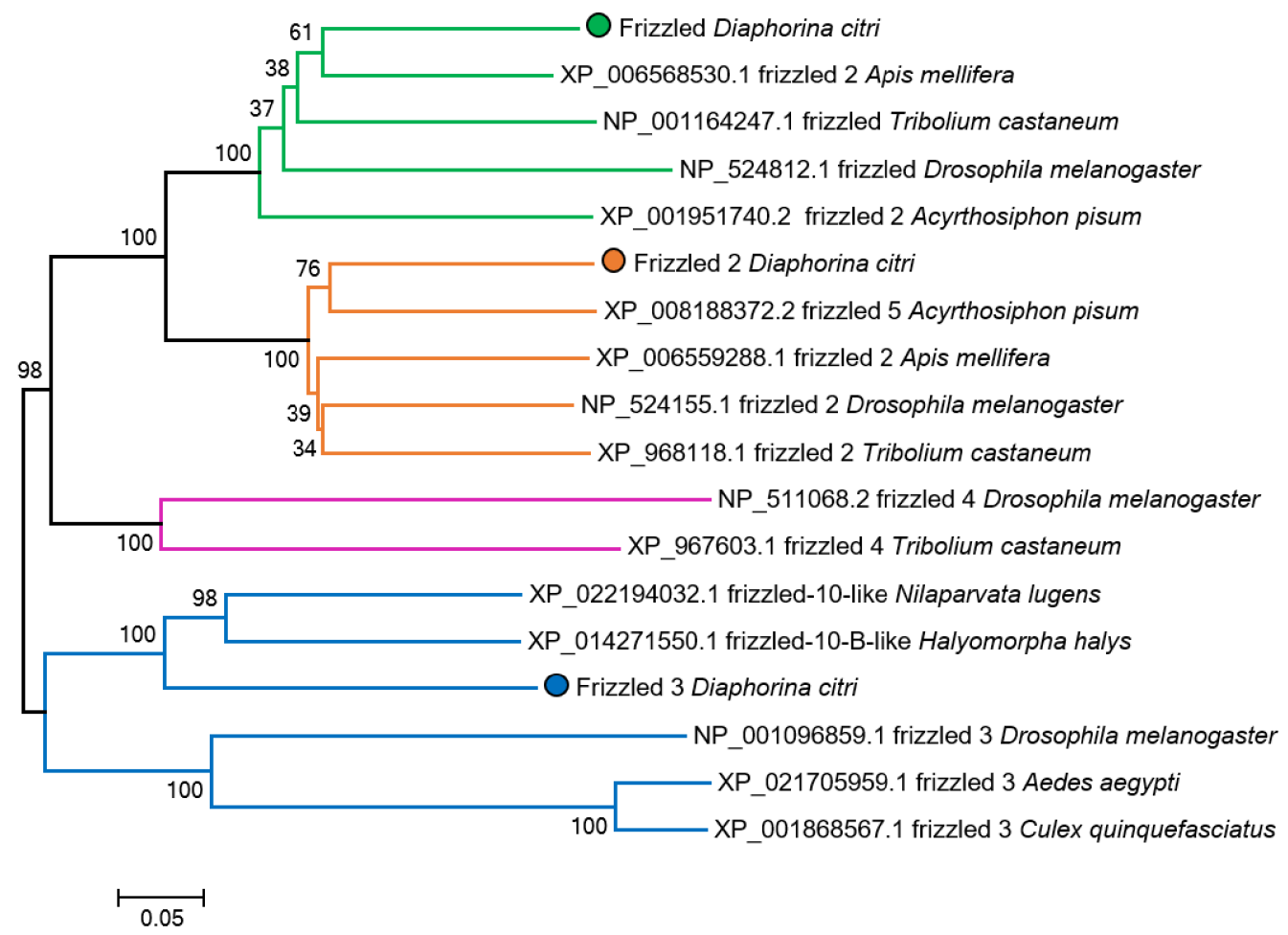

Figure 8. Neighbor-joining tree of insect Frizzled protein sequences. Proteins grouped in the Frizzled 1 subfamily are highlighted in green, Frizzled 2 in orange, Frizzled 3 in blue, and Frizzled 4 in magenta. Circles indicate the $D$. citri sequences. Ortholog sequences were collected from the NCBI protein database [18]. Some NCBI sequences (such as XP_006568530.1,XP_008188372.2, and XP_022194032.1) may have numeric labels derived from computational predictions that do not reflect sequence or functional similarity. Analysis was performed using MEGA7 [23].

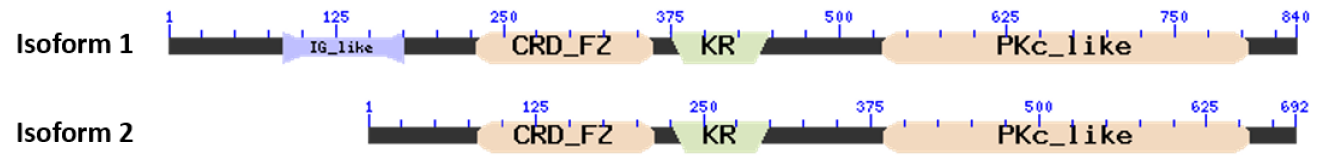

Figure 9. Domain comparison of ROR1 isoforms. The immunoglobulin domain (IG_like) is present in isoform 1 . Other shared domains include a cysteine-rich frizzled domain (CRD_FZ), a Kringle domain (KR), and a protein kinase catalytic domain (PKc_like). Domains were calculated and visualized using the NCBI Conserved Domain Architecture Retrieval Tool (CDART).

\section{DATA AVAILABILITY}

Our annotation and gene curation workflow is described by Shippy et al. [17]. The Diaphorina citri genome assembly, official gene sets, and transcriptome data are accessible on the Citrus Greening website [50]. All accessions for genes used for phylogentic analysis are provided within this report (Tables 2, 3, Figure 8). We have included the Newick and Multiple Sequence Alignment files used to construct the Wnt neighbor-joining phylogenetic tree and other data is available in the GigaScience GigaDB repository [51]. 


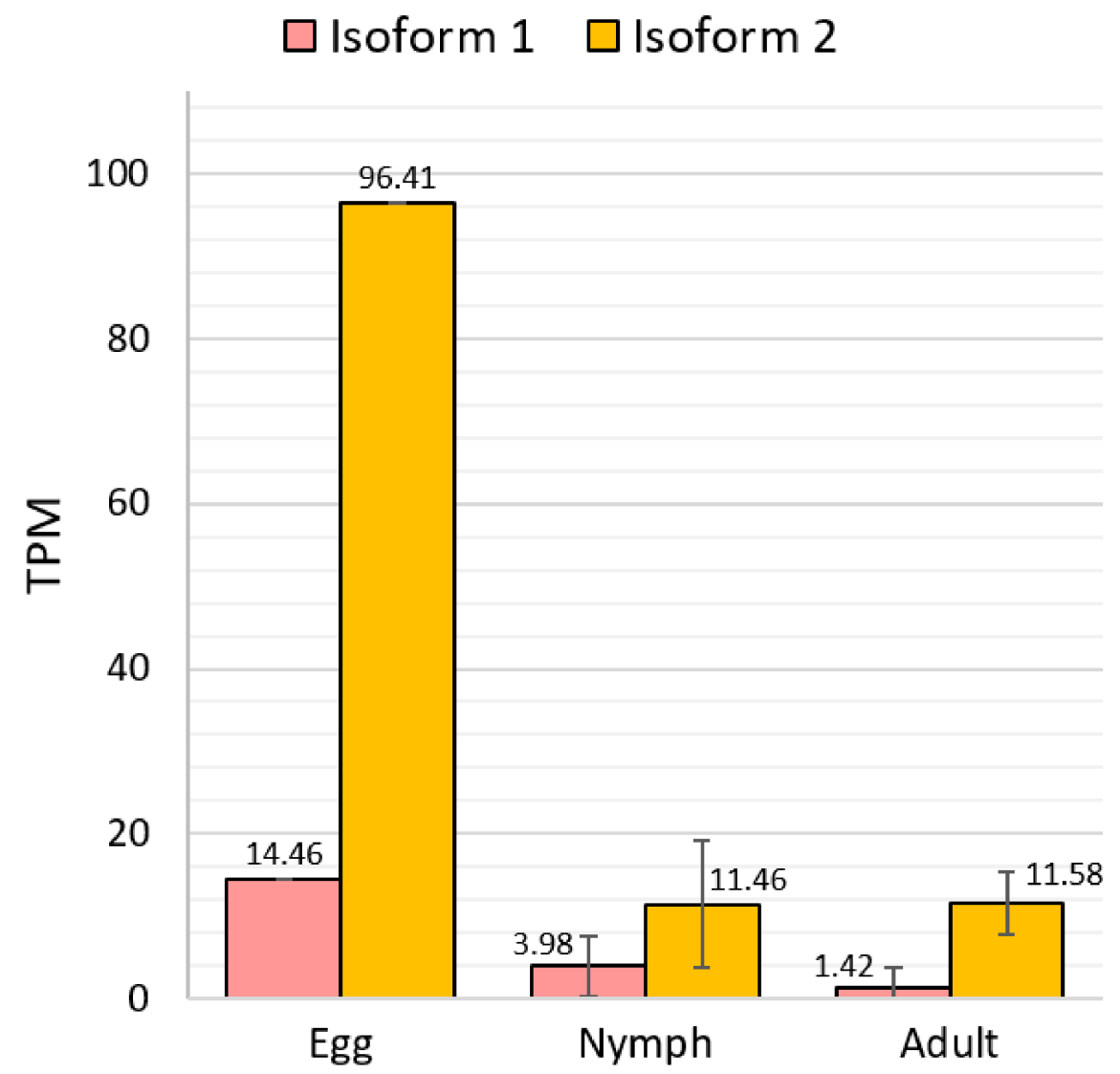

Figure 10. Expression of ROR1 isoforms in egg, nymph and adult $D$. citri. Pink bars indicate the average transcript levels for isoform 1 (Dcitr05g14430.1.1), and orange bars indicate the average transcript levels for isoform 2 (Dcitr05g14430.1.2). Note: only one egg sample was used for comparison. Egg transcripts from the whole egg (one sample), nymph transcripts from the whole body (six samples), and adult transcripts from the whole body, abdomen, and thorax (14 samples) are shown. Expression values shown in transcripts per million (TPM). Data labels note the average TPM. Standard deviation of samples, when available, is shown by error bars. RNA-seq data was collected from CGEN [48].

\section{EDITOR'S NOTE}

This article is one of a series of Data Releases crediting the outputs of a student-focused and community-driven manual annotation project, curating gene models and - if required correcting assembly anomalies, for the Diaphorina citri genome project [46].

\section{DECLARATIONS}

\section{LIST OF ABBREVIATIONS}

CGEN: Citrus Greening Expression Network; HLB: Huanglongbing; MCOT: Maker, Cufflinks, Oases, and Trinity; NCBI: National Center for Biotechnology Information

\section{ETHICAL APPROVAL}

Not applicable.

\section{CONSENT FOR PUBLICATION}

Not applicable. 


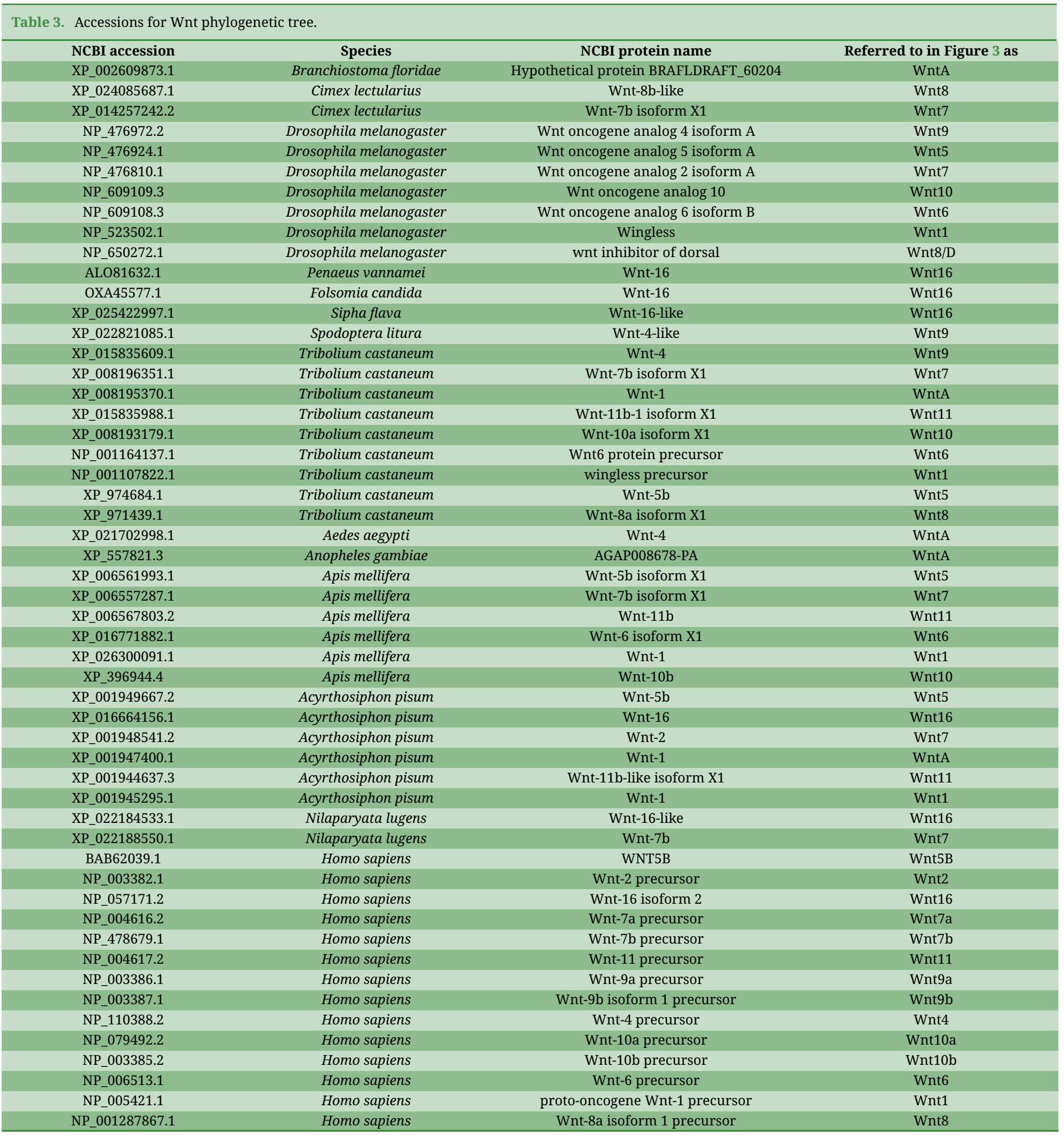




\section{COMPETING INTERESTS}

The authors declare that they have no competing interests.

\section{AUTHORS" CONTRIBUTIONS}

WBH, SJB, TD and LAM conceptualized the study; TD, SS, TDS and SJB supervised the study; SJB, TD, SS, and LAM contributed to project administration; CV, MR, and RN conducted investigation; PH, MF-G, and SS contributed to software development; SS, TDS, PH, and MF-G developed methodology; SJB, TD, WBH, and LAM acquired funding; CV prepared and wrote the original draft; TD, SS, TDS, and SJB reviewed and edited the draft.

\section{FUNDING}

This work was supported by USDA-NIFA grants 2015-70016-23028, HSI 1300394 and 2020-70029-33199.

\section{ACKNOWLEDGEMENTS}

We thank Alistair McGregor and Joshua Benoit for valuable discussions.

\section{REFERENCES}

1 Bové JM, Huanglongbing: a destructive, newly-emerging, century-old disease of citrus. J. Plant Pathol., 2006; 88: 7-37.

2 Gottwald TR, Current epidemiological understanding of citrus Huanglongbing. Annu. Rev. Phytopathol., 2010; 48: 119-139.

3 Hunter WB, Gonzalez MT, Tomich J, BAPC-assisted CRISPR/Cas9 system: targeted delivery into adult ovaries for heritable germline gene editing (Arthropoda: Hemiptera). BioRxiv. Cold Spring Harbor Laboratory; 2018; https://doi.org/10.1101/478743.

4 Hunter WB et al. Advances in RNA suppression of the Asian citrus psyllid vector and bacteria (Huanglongbing pathosystem). In: Qureshi JA, Stansly PA (eds), Asian Citrus Psyllid: Biology, Ecology and Management of the Huanglongbing Vector. Wallingford: CABI 2020; p. 258.

5 Chen XD, Stelinski LL, Resistance management for Asian Citrus Psyllid, Diaphorina citri Kuwayama, in Florida. Insects, 2017; 8: 103.

6 Kanga LHB, Eason J, Haseeb M, Qureshi J, Stansly P, Monitoring for insecticide resistance in Asian Citrus Psyllid (Hemiptera: Psyllidae) populations in Florida. J. Econ. Entomol., 2016; 109: 832-836.

7 Pardo S et al. Insecticide resistance of adults and nymphs of Asian citrus psyllid populations from Apatzingán Valley, Mexico. Pest Manag. Sci., 2018; 74: 135-140.

8 Holstein TW, The evolution of the Wnt pathway. Cold Spring Harb. Perspect. Biol., 2012; 4: a007922.

9 Nusse R et al. Wnt signaling and stem cell control. Cold Spring Harb. Symp. Quant. Biol., 2008; 73 : 59-66.

10 Nusse R., The Wnt gene homepage. 2020. http://web.stanford.edu/group/nusselab/cgi-bin/wnt/. Accessed 18 December 2020.

11 Klingensmith J, Nusse R, Signaling by wingless in Drosophila. Dev. Biol., 1994; 166: 396-414.

12 Bhanot P, Fish M, Jemison JA, Nusse R, Nathans J, Cadigan KM, Frizzled and Dfrizzled-2 function as redundant receptors for Wingless during Drosophila embryonic development. Development, 1999; 126: $4175-4186$.

13 Kadowaki T, Wilder E, Klingensmith J, Zachary K, Perrimon N, The segment polarity gene porcupine encodes a putative multitransmembrane protein involved in Wingless processing. Genes Dev., 1996; 10: 3116-3128.

14 Bolognesi R, Farzana L, Fischer TD, Brown SJ, Multiple Wnt genes are required for segmentation in the short-germ embryo of Tribolium castaneum. Curr. Biol., 2008; 18: 1624-1629.

15 Beermann A, Prühs R, Lutz R, Schröder R, A context-dependent combination of Wnt receptors controls axis elongation and leg development in a short germ insect. Development, 2011; 138: 2793-2805. 
16 Suzuki T, Trush O, Yasugi T, Takayama R, Sato M, Wnt signaling specifies anteroposterior progenitor zone identity in the Drosophila visual center. J. Neurosci., 2016; 36: 6503-6513.

17 Shippy TD et al. Annotating genes in Diaphorina citri genome version 3. protocols.io. 2020; https://dx.doi.org/10.17504/protocols.io.bniimcce.

18 NCBI Protein Database. https://www.ncbi.nlm.nih.gov/protein/.

19 Saha S et al. Improved annotation of the insect vector of citrus greening disease: biocuration by a diverse genomics community. Database. 2017; https://doi.org/10.1093/database/bax032.

20 Janssen $\mathbf{R}$ et al. Conservation, loss, and redeployment of Wnt ligands in protostomes: implications for understanding the evolution of segment formation. BMC Evol. Biol., 2010; 10: 374.

21 Bao R, Fischer T, Bolognesi R, Brown SJ, Friedrich M, Parallel duplication and partial subfunctionalization of $\beta$-catenin/armadillo during insect evolution. Mol. Biol. Evol., 2012; 29: 647-662.

22 Shigenobu S et al. Comprehensive survey of developmental genes in the pea aphid, Acyrthosiphon pisum: frequent lineage-specific duplications and losses of developmental genes. Insect Mol. Biol., 2010; 19: 47-62.

23 Kumar S, Stecher G, Tamura K, MEGA7: Molecular evolutionary genetics analysis version 7.0 for bigger datasets. Mol. Biol. Evol., 2016; 33: 1870-1874.

24 Thurmond J et al. FlyBase 2.0: the next generation. Nucleic Acids Res., 2019; 47: D759-D765.

25 Tribolium Genome Sequencing Consortium. The genome of the model beetle and pest Tribolium castaneum. Nature, 2008; 452: 949-955.

26 Kawamoto $\mathbf{M}$ et al. High-quality genome assembly of the silkworm, Bombyx mori. Insect Biochem. Mol. Biol., 2019; 107: 53-62.

27 Elsik CG et al. Finding the missing honey bee genes: lessons learned from a genome upgrade. BMC Genomics, 2014; 15: 86.

28 Werren JH et al. Functional and evolutionary insights from the genomes of three parasitoid Nasonia species. Science, 2010; 327: 343-348.

29 International Aphid Genomics Consortium. Genome sequence of the pea aphid Acyrthosiphon pisum. PLoS Biol., 2010; 8: e1000313.

30 Xue J et al. Genomes of the rice pest brown planthopper and its endosymbionts reveal complex complementary contributions for host adaptation. Genome Biol., 2014; 15: 521.

31 Ma W et al. Chromosomal-level genomes of three rice planthoppers provide new insights into sex chromosome evolution. Mol. Ecol. Resour., 2021; 21: 226-237.

32 O'Leary NA et al. Reference sequence (RefSeq) database at NCBI: current status, taxonomic expansion, and functional annotation. Nucleic Acids Res., 2016; 44: D733-D745.

33 Sparks ME et al. Brown marmorated stink bug, Halyomorpha halys (Stål), genome: putative underpinnings of polyphagy, insecticide resistance potential and biology of a top worldwide pest. BMC Genomics, 2020; 21: 227.

34 Benoit JB et al. Unique features of a global human ectoparasite identified through sequencing of the bed bug genome. Nat. Commun., 2016; 7: 10165.

35 Matthews BJ et al. Improved reference genome of Aedes aegypti informs arbovirus vector control. Nature, 2018; 563: 501-507.

36 Holt RA et al. The genome sequence of the malaria mosquito Anopheles gambiae. Science, 2002; 298 : 129-149.

37 Putnam NH et al. The amphioxus genome and the evolution of the chordate karyotype. Nature, 2008; 453: 1064-1071.

38 Zhang S et al. Molecular cloning, characterization and expression analysis of Wnt4, Wnt5, Wnt6, Wnt7, Wnt10 and Wnt16 from Litopenaeus vannamei. Fish Shellfish Immunol., 2016; 54: 445-455.

39 Faddeeva-Vakhrusheva A et al. Coping with living in the soil: the genome of the parthenogenetic springtail Folsomia candida. BMC Genomics, 2017; 18: 493.

40 Cheng T et al. Genomic adaptation to polyphagy and insecticides in a major East Asian noctuid pest. Nat. Ecol. Evol., 2017; 1: 1747-1756.

41 Panfilio KA et al. Molecular evolutionary trends and feeding ecology diversification in the Hemiptera, anchored by the milkweed bug genome. Genome Biol., 2019; 20: 64 . 
42 Bolognesi R et al. Tribolium Wnts: evidence for a larger repertoire in insects with overlapping expression patterns that suggest multiple redundant functions in embryogenesis. Dev. Genes Evol., 2008; 218: 193-202.

43 Doumpas N, Jékely G, Teleman AA, Wnt6 is required for maxillary palp formation in Drosophila. BMC Biol., 2013; 11: 104.

44 Sullivan JC, Ryan JF, Mullikin JC, Finnerty JR, Conserved and novel Wnt clusters in the basal eumetazoan Nematostella vectensis. Dev. Genes Evol., 2007; 217: 235-239.

45 Nusse R, An ancient cluster of Wnt paralogues. Trends Genet., 2001; 17: 443.

46 Hosmani PS et al. Chromosomal length reference assembly for Diaphorina citri using single-molecule sequencing and $\mathrm{Hi}-\mathrm{C}$ proximity ligation with manually curated genes in developmental, structural and immune pathways. BioRxiv. Cold Spring Harbor Laboratory; 2019; https://doi.org/10.1101/869685.

47 Reese J et al. Characterization of the Asian Citrus Psyllid transcriptome. J. Genomics, 2014; 2: 54-58.

48 Flores-Gonzalez $\mathbf{M}$ et al. Citrusgreening.org: An open access and integrated systems biology portal for the Huanglongbing (HLB) disease complex. BioRxiv. 2019; https://doi.org/10.1101/868364.

49 Citrus Greening Solutions. Annotation of psyllid genome. 2018. https://citrusgreening.org/annotation/index. Accessed 18 December 2020.

50 Citrus Greening Solution. https://citrusgreening.org/. Accessed 18 December 2020.

51 Vosburg C, Reynolds M, Noel R, Shippy TD, Hosmani PS, Flores-Gonzalez M, Mueller LA, Hunter WB, Brown SJ, D’Elia T, Saha S, Supporting data for “Utilizing a chromosomal-length genome assembly to annotate the Wnt signaling pathway in the Asian citrus psyllid, Diaphorina citri”. GigaScience Database. 2021; http://dx.doi.org/10.5524/100898. 